\title{
Formação em Nutrição em Saúde Coletiva na Universidade Federal de Santa Catarina: reflexões sobre o processo de ensino para fortalecer o Sistema Único de Saúde
}

\author{
Professional Qualification in Public Health Nutrition at \\ Universidade Federal de Santa Catarina: \\ Reflections on the teaching process to strengthen \\ the Brazilian Unified Health System
}

Janaina DAS NEVES 1

Anete Araújo de SOUSA

Francisco de Assis Guedes de VASCONCELOS'

O objetivo do artigo é relatar a experiência do processo de implantação do projeto pedagógico do Curso de Graduação em Nutrição da Universidade Federal de Santa Catarina visando ao atendimento das Diretrizes Curriculares dos Cursos de Nutrição de 2001, com ênfase na formação em Saúde Coletiva. Trata-se de artigo descritivo que procura refletir sobre a experiência da reestruturação curricular implantada em 2009. A reestruturação curricular proposta pelo Núcleo Docente Estruturante contou com assessoria externa, parceria das Secretarias de Saúde e de Educação de Florianópolis e financiamento do Ministério da Saúde, tendo por base as avaliações realizadas em fóruns com participação de docentes, discentes e nutricionistas. O projeto pedagógico do Curso de Graduação em Nutrição da Universidade Federal de Santa Catarina pautou-se pelos princípios do Direito Humano à Alimentação Adequada, da Segurança Alimentar e Nutricional e da promoção, proteção e recuperação da saúde. O currículo contempla os níveis de complexidade de atenção à saúde do Sistema Único de Saúde, com integração entre disciplinas que desenvolvem metodologias ativas, durante as aulas teóricas e práticas. Outros cenários de prática são: unidades educativas, locais de produção e comercialização de alimentos e unidades de alimentação e nutrição. Os coordenadores pedagógicos realizam interlocução entre secretarias, profissionais, docentes e discentes. O currículo tem sido avaliado e reestruturado continuamente. Apesar dos avanços, são apontados como desafios: a integração ensino-serviço, o número reduzido de nutricionistas nos cenários de prática, a estrutura disciplinar e o grande número de estudantes nas turmas práticas.

Termos de indexação: Ensino superior. Nutrição. Saúde coletiva. Sistema Único de Saúde.

\footnotetext{
1 Universidade Federal de Santa Catarina, Centro de Ciências da Saúde, Departamento de Nutrição. Campus Universitário Trindade, Caixa Postal 476, 88040-900, Florianópolis, SC, Brasil. Correspondência para/Correspondence to: FAG VASCONCELOS. E-mail:<f.vasconcelos@ufsc.br>.
} 


\title{
A B S T R A C T
}

\begin{abstract}
The aim of the paper is to report the experience of implementing the pedagogical project of the Undergraduate Course in Nutrition of Universidade Federal de Santa Catarina, Brazil, aiming to meet the Curriculum Guidelines for Nutrition Courses 2001, with emphasis on the formation process in public health. This is a descriptive article that seeks to reflect on the experience of curriculum restructuring implemented in 2009. The pedagogical project of the Undergraduate Course in Nutrition of Universidade Federal de Santa Catarina, was guided by the principles of the Human Right to Adequate Food, Food and Nutritional Security and the promotion, protection and recovery health. The curriculum restructuring proposed by the structuring teaching core receives external assessory, a partnership of the Departments of Health and Education of Florianópolis, Brazil, and funding from the Brazilian Ministry of Health, based on the assessments carried out on forums with participation of teachers, students and nutritionists. The curriculum comprises the levels of complexity of healthcare of the Brazilian Unified Health System, with integration between disciplines who develop active methods during the theoretical and practical classes. Other practice scenarios are: educational units, establishments of production and marketing of food, and food and nutrition units. The pedagogical coordinators conduct dialogue between Departments, professionals, teachers and students. The curriculum has been evaluated and restructured continuously. Despite the advances, challenges are noted as: A teaching-service integration, the reduced number of nutritionists to practical, disciplinary structure and large number of students in practical classes.
\end{abstract}

Indexing terms: Higher education. Nutrition. Public health. Brazilian Unified Health System.

\section{N T R O D U ÇÃ O}

A promulgação da Lei de Diretrizes e Bases da Educação Nacional (LDB) em dezembro de 1996', tem sido identificada como o evento propulsor da redefinição do sistema educacional brasileiro, inclusive, da ampliação do debate em torno da formação profissional do nutricionista, da instituição das diretrizes para reformulação curricular e, simultaneamente, da intensa ampliação do número de cursos de graduação em Nutrição no Brasil|2-7.

Na sequência, entre 1997 a agosto de 2001, ocorreu o processo de debate e construção das Diretrizes Curriculares Nacionais (DCN) dos cursos de graduação, o qual culminou na publicação, em 7 de novembro de 2001, da Resolução $n^{\circ} 5$ do Conselho Nacional da Educação/Câmara de Educação Superior (CNE/CES) do Ministério da Educação que instituiu as DCN do Curso de Graduação em Nutrição\&.

A partir das Diretrizes Curriculares Nacionais de $2001^{8}$, o debate sobre a formação do nutricionista tem sido pautado na necessidade das instituições formadoras centrarem esforços para o desenvolvimento de práticas de ensino voltadas para o fortalecimento do Sistema Único de Saúde (SUS) e, portanto, na formação de profissionais capazes de prestarem atendimento integral e humanizado, de trabalhar em equipes multidisciplinares e de compreender melhor a realidade em que vive a população. Além disso, dentro da compreensão dos diferentes níveis de complexidade da atenção à saúde, é responsabilidade da formação preparar profissionais capazes de realizarem no cotidiano de sua prática profissional, ações que visem à promoção, proteção e recuperação em nível individual e coletivo.

Esse debate suscitou a publicação nos últimos cinco anos (2009 a abril de 2014) de considerável número de artigos $(n=22)$ sobre o processo de formação e de atuação profissional do nutricionista no Brasil| $2-7,9-24$.

Em relação ao processo específico de formação do nutricionista na área de Saúde Coletiva, foram identificados sete artigos publicados nos últimos cinco anos $23,6,7,9,10,18$, observando-se distintos desenhos e enfoques analíticos entre estes sete artigos, desde aqueles com abordagens teórico-conceituais ${ }^{2,3,7}$, àqueles centrados em abordagens empíricas ${ }^{6,9,10,18}$, sejam elas com enfoque qualitativo $0^{9,10}$, quantitativo ${ }^{6}$ ou quantiqualitativo $^{18}$.

Sobre o processo de atuação do nutricionista na área de Saúde Coletiva, foram iden- 
tificados 15 artigos publicados nos últimos cinco anos $4,5,11,17,19-24$, os quais se distribuem em abordagens teórico-conceituais $(n=7)^{4,5,11-13,15,19}$ e abordagens empíricas $(n=8)^{14,16,17,20-24}$. Estes 15 artigos sobre a atuação do nutricionista no campo da Saúde Coletiva tomaram como objetos de análises distintas temáticas, tais como aspectos histórico-

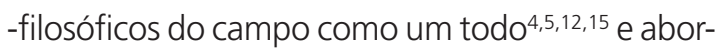
dagens sobre subáreas específicas da atuação neste campo, tais como a atuação na Atenção Básica ${ }^{14,17,24}$, nos Núcleos de Apoio à Saúde da Família (NASF) ${ }^{13,16}$, na Alimentação Escolar 11,20,22, na Segurança Alimentar e Nutricional $(\mathrm{SAN})^{23}$ e na Educação Alimentar e Nutricional no âmbito das Políticas Públicas ${ }^{19,21}$.

Embora se identifique um interesse emergente de investigação dessa temática nos últimos cinco anos, pelo mapeamento desse estado da arte consideram-se ainda escassos os estudos sobre a formação profissional do nutricionista como um todo (englobando as sete distintas áreas de atuação estabelecidas pelo Conselho Federal de Nutricionistas [CFN] ${ }^{25}$, as quais caracterizam o processo de formação generalista). Consideram-se também escassos os estudos sobre formação específica do nutricionista em Saúde Coletiva, área de atuação definida pelo CFN como o conjunto de atividades de alimentação e nutrição realizadas em políticas e programas institucionais, de atenção básica e de vigilância sanitária ${ }^{25}$. São escassos também os estudos sobre relatos e/ou avaliações de processos de implantação dos projetos pedagógicos dos cursos de graduação em Nutrição posteriores às DCN de 2001. Nos últimos cinco anos, nenhuma publicação sobre relatos e/ou avaliações de processos de implantação dos projetos pedagógicos dos cursos de graduação em Nutrição pós DCN de 2001 foi localizada. Foi identificado apenas um estudo desta natureza, publicado em $2005^{26}$.

Nesse sentido, o presente artigo tem por objetivo relatar a experiência do processo de implantação do projeto pedagógico do Curso de Graduação em Nutrição da Universidade Federal de Santa Catarina (UFSC) visando ao atendimento das DCN de $2001^{8}$, com ênfase na formação em Saúde Coletiva.

Este artigo encontra-se estruturado em três seções. Na primeira procuramos relatar um breve histórico do Curso de Graduação em Nutrição da UFSC. Na segunda relatamos como ocorre a formação em Nutrição em Saúde Coletiva na UFSC, com ênfase nas atividades práticas. E na terceira discutimos os desafios para a formação em Nutrição em Saúde Coletiva no âmbito da UFSC.

\section{Breve histórico do Curso de Graduação em Nutrição da UFSC}

Em Santa Catarina, conforme já apontado em trabalhos anteriores ${ }^{27,28}$, a história do processo de formação profissional do nutricionista se encontra vinculada à criação do Curso de Graduação em Nutrição da UFSC. Este curso foi criado por meio da Portaria n 384/Gabinete do Reitor UFSC, de 10 de agosto de 1979, e implantado no primeiro semestre letivo de 1980. Em 22 de fevereiro de 1985, por meio do Parecer no 109/85 e da Portaria Ministerial $n^{\circ} 249$, de 25 de março de 1985, obteve o reconhecimento pelo Ministério da Educação e Cultura. A primeira turma de egressos deste curso, composta por 20 nutricionistas, colou grau em dezembro de $1983^{27}$. Até novembro de 2014, ao completar 34 anos de implantação, 30 turmas haviam colado grau, totalizando 829 nutricionistas egressos da UFSC ${ }^{29}$.

Ao longo de seus 34 anos, o Curso desenvolveu sistematicamente avaliações de sua prática pedagógica, buscando aprimorar a qualidade do ensino na Nutrição. Ressalta-se que a emergência do debate sobre as mudanças na formação não foi uma constatação isolada do Curso da UFSC, no cenário nacional esse debate emergiu de forma ampliada para todo ensino superior. No caso das profissões da área da saúde, dentre elas a de nutricionista, as críticas e avaliações sobre o processo de formação apontavam, em geral, os seguintes pontos comuns: 1) predomínio da visão biológica do homem; 2) fragmentação do conhecimento 
e, portanto, da unidade do homem; 3) ênfase na análise e na resolução da enfermidade, e não no processo saúde-doença; 4) divisão da teoria e da prática, do básico e do profissionalizante (com ênfase no atendimento clínico); 5) predomínio das especialidades, com tendência à transferência da responsabilidade da formação para a pós-graduação; 6) valorização crescente da clínica, consolidando a atenção/prática curativa de alta complexidade como eixo da formação profissional; 7) exaltação do curativo e postergação da importância da promoção da saúde e da prevenção das doenças; 8) posição secundária das Ciências Sociais na formação; 9) alta valorização do exercício liberal e da competência, no futuro exercício profissional; 10) defasagem entre os conteúdos utilizados no processo formativo dos profissionais e as reais necessidades de saúde da população; e 11) escassez ou desenvolvimento insuficiente do raciocínio científico necessário à pesquisa ${ }^{2,3,6,30}$.

Desde os primeiros anos de 1990, a UFSC desenvolve experiência institucionalizada de articulação com o SUS, nos níveis de graduação, pós-graduação e educação permanente, por meio do Polo de Educação Permanente para o SUS e do Programa de Articulação da Rede Docente Assistencial (RDA) (parceria entre UFSC, Secretaria Municipal de Saúde de Florianópolis e Hospital Universitário) ${ }^{31}$.

Uma vez aprovadas as DCN de 20018, o Curso vem realizando encontros anuais, envolvendo docentes e discentes e contando com contribuições de educadores e nutricionistas das Redes de Saúde e Educação do Município de Florianópolis com o objetivo de repensar a formação e definir a concepção do currículo.

A partir de 2002, o Curso e o Departamento de Nutrição da UFSC formularam um projeto com o objetivo de realizar avaliações curriculares e, ao mesmo tempo, buscar o desenvolvimento de projetos pedagógicos que possibilitem uma visão inter e transdisciplinar do conhecimento em Nutrição. Foi criado o "Fórum de Nutrição", espaço de discussão composto pelo Centro
Acadêmico de Nutrição, Empresa Junior de Nutrição, Programa de Educação Tutorial (PET da Nutrição) e Curso/Departamento de Nutrição da UFSC. O Fórum propiciou a reflexão sobre a avaliação do currículo, a elaboração de propostas de mudanças curriculares e a consolidação da Semana da Nutrição da UFSC como um momento de reflexão e debates sobre a formação de nutricionistas.

Em 2003, durante a "IV Semana de Nutrição da UFSC", foram elaboradas propostas a respeito de aspectos relacionados às mudanças curriculares, tais como: 1) criar pré-estágios por fase ou ano, como um espaço de formação que relacione a teoria e a prática; 2) criar os conselhos de fase, composto por representantes de turmas e professores, cuja função seria discutir planos de ensino, conteúdos, metodologias, avaliações e frequência; 3) pensar no currículo como um espaço de produção e integração do conhecimento; 4) repensar a primeira fase do curso garantindo o espaço de acolhimento do estudante; 5) propor mais momentos de reflexão e discussão sobre o ensino, proporcionando a reciclagem pedagógica constante; e 6) oferecer aos acadêmicos do Curso um maior número de disciplinas optativas em diferentes áreas do conhecimento e avaliar a proposta de inserção do Trabalho de Conclusão de Curso (TCC) no último ano do Curso.

Em 2005 foi formada a Comissão do Projeto Político Pedagógico (PPP) formalmente instituída por meio da Portaria $n^{\circ} 132$ do Centro de Ciências da Saúde da UFSC de 23 de novembro de 2006. Durante o ano de 2006 a comissão promoveu encontros para sensibilização de professores e estudantes para as mudanças curriculares necessárias para adequação do Curso às DCN de 2001․ A "VIII Semana da Nutrição da UFSC", realizada em 2007, propiciou o debate com profissionais, ligados ao ensino nas áreas da Saúde e da Educação para subsidiar as mudanças necessárias na formação dos estudantes de Nutrição.

Com base neste processo e nas recomendações geradas nas "Semanas de Nutrição", a Comissão do PPP elaborou a primeira versão de 
uma nova matriz curricular para o Curso de Nutrição da UFSC, que foi submetida à apreciação em 2008 durante "IX Semana da Nutrição".

O Projeto Pedagógico do Curso foi aprovado pelas instâncias deliberativas em 15 de dezembro de 2009 por meio da Portaria n 397 da Pró-Reitoria de Ensino de Graduação. Tal projeto tem como objetivo reorientar o processo de formação do nutricionista do Curso da UFSC para o desenvolvimento de ações de promoção e proteção da saúde, em nível individual, comunitário e populacional, considerando os conceitos de $\mathrm{SAN}^{32}$ e Direito Humano à Alimentação Adequada (DHAA) ${ }^{33}$, conjugados aos da interdisciplinariedade, humanismo, flexibilidade e ética, no processo ensino-aprendizagem ${ }^{8,30}$.

Estes elementos embasaram a concepção do perfil do egresso e os objetivos do Curso, aprovados na "IX Semana de Nutrição", em 2008. O perfil do egresso foi definido como um profissional cidadão, com formação generalista, autônomo na busca do conhecimento, sensível e crítico em relação à realidade social, econômica, política e cultura, percebendo o ser humano e o mundo em sua integralidade, capaz de atuar como agente de transformação, trabalhando interdisciplinarmente de forma comprometida, ética e sustentável, em todos os campos de conhecimento em que a alimentação e a nutrição se apresentem fundamentais para a promoção, manutenção e recuperação da saúde de indivíduos e coletividade. Por sua vez, os objetivos do Curso foram assim delineados:

Desenvolver nos estudantes a capacidade de articular os conhecimentos biológicos, políticos, econômicos e sociais em todas as áreas em que a alimentação e a nutrição são fundamentais para preservar, promover e recuperar a saúde de indivíduos e grupos populacionais, a partir de instrumental técnico e científico ${ }^{34}$ (p.1).

Desenvolver atividades no campo da nutrição e alimentação humana, com percepção crítica da realidade social, econômica, cultural e política, visando à garantia da Segurança Alimentar e Nutricional e do Direito Humano à Alimentação Adequada em nível individual e coletivo 34 (p.1).

Portanto, o novo currículo do Curso de Nutrição da UFSC teve seu início efetivado no primeiro semestre letivo de $2009^{34}$. Ressalta-se que a primeira turma pós reforma curricular de 2009, atendendo aos requisitos das DCN de $2001^{8}$, composta por 30 nutricionistas, colou grau em 13 de dezembro de 2013.

A efetivação da reestruturação curricular de 2009 coincide com a aprovação da participação do curso no Programa Nacional de Reorientação da Formação Profissional em Saúde (Pró-Saúde II) ${ }^{30}$, o que intensificou a integração com os nutricionistas que atuam na atenção básica à saúde. Desde então são realizadas reuniões periódicas, com a presença destes profissionais e daqueles que atuam no programa municipal de alimentação escolar, as quais têm por objetivos definir os territórios que servirão de cenário de prática, bem como planejar, organizar e avaliar as atividades práticas das distintas fases do Curso.

Desde a sua implantação em 2009, o currículo tem sido acompanhado continuamente por grupo de professores que compõem o Núcleo Docente Estruturante (NDE) e pelos diferentes atores e parceiros que compõem o Curso de Nutrição, por meio de discussões nos chamados fóruns internos (docentes e discentes do Curso de Nutrição) e externos (nutricionistas da atenção básica e alimentação escolar) do Curso de Nutrição. Dessa forma, o Curso de Nutrição da UFSC apresenta características diferenciadas, principalmente no tocante à formação em Nutrição Coletiva, conforme será apresentado na seção a seguir.

\section{A formação em Nutrição em Saúde Coletiva na UFSC}

A área de conhecimento de Nutrição em Saúde Coletiva, assim como as demais áreas que 
compõem o Departamento de Nutrição da UFSC (Nutrição Clínica, Nutrição em Unidades de Alimentação e Nutrição e Nutrição Básica e Experimental), é organizada de maneira a atender a formação de um profissional generalista com base nos pressupostos relatados e contemplados no perfil do egresso. Para isto, os conhecimentos específicos para a formação em Nutrição em Saúde Coletiva exigiu a concepção de um Projeto Pedagógico que ense-jasse a formação nessa área com as seguintes características:

- Estruturação de disciplinas interáreas que possibilitem articulações com base em um objeto e práticas integradoras e promovam a verticalização do conhecimento;

- Implementação de processos de ensino-aprendizagem que promovam o compartilhamento e construção de conhecimentos, priorizando metodologias ativas;

- Inserção em cenários de prática que contemplem a complexidade da atenção em saúde (da atenção básica à alta complexidade); que possibilitem a atuação na rede de ensino básico (da educação infantil ao ensino médio) e em locais que compõem o sistema alimentar do território (locais de produção de alimentos, estabelecimentos de compra e de consumo da alimentação fora de casa);

- Definição de coordenações pedagógicas (coordenador de fase) do primeiro ao quarto ano do Curso por área de conhecimento para interlocução entre Secretarias, profissionais, docentes e discentes; e

- Articulação com profissionais das Redes de Saúde e de Educação "Fórum de Integração Ensino-Serviço" para acompanhamento e avaliação das atividades práticas em cinco territórios situados em Distritos Sanitários do município de Florianópolis.

As reuniões de fase são estratégias que auxiliam na integração dos conhecimentos trabalhados. Tais reuniões têm por objetivo possibilitar um panorama das disciplinas em seus encontros teóricos e atividades práticas, contribuindo para a verticalização dos conteúdos entre as fases curriculares ${ }^{35-38}$.

\section{A verticalização dos conteúdos em Nutrição em Saúde Coletiva na UFSC}

As disciplinas que fortalecem o entendimento da atuação do nutricionista em saúde coletiva (atenção básica, políticas e programas institucionais e vigilância sanitária) $)^{8,25,30}$ estão distribuídas ao longo dos cinco anos de integralização curricular. No Anexo encontram-se listadas estas disciplinas.

A matriz curricular do Curso de Nutrição da UFSC foi estruturada de forma que as disciplinas e seus respectivos conteúdos (ementas) respondam a um objeto integrador. O objeto integrador consiste em um tema problematizador que norteia as atividades práticas articuladas e discussões teóricas a cada ano/semestre do Curso. Destaca-se que o Curso não possui uma estrutura modular, mas, com base no tema problematizador, as disciplinas foram alocadas de forma a responder a uma lógica de verticalização de conhecimentos com base na complexidade da atenção em saúde, conforme descrição a seguir ${ }^{8,35-38}$.

- $1^{\circ}$ ano: O território e o diagnóstico em saúde, alimentação e nutrição: O primeiro ano do Curso está estruturado de maneira a apresentar ao estudante a porta de entrada do SUS, a atenção básica. O cenário de prática de referência é o Centro de Saúde da Família (CSF).

$\mathrm{Na}$ atividade prática da primeira fase, denominada de territorialização, os estudantes conhecem a estrutura física de um CSF, contam com a parceria de Agentes Comunitários de Saúde (ACS) para conhecer o território e entram em contato com Equipes de Saúde da Família (ESF) e os profissionais dos NASF.

Destaca-se que as atividades práticas contam com professores orientadores de todas as áreas de conhecimento do Departamento de Nu- 
trição, na expectativa de que o processo de territorialização possibilite aos docentes e discentes um olhar atento sobre os instrumentos necessários para a formação em cada área.

Durante a atividade prática da segunda fase, os estudantes retornam aos mesmos territórios para identificação das políticas e programas públicos relacionados à saúde, alimentação e nutrição. As informações sobre o funcionamento dos programas e ações de alimentação e nutrição são obtidas com os coordenadores dos CSF e diretores de unidades educativas públicas, privadas e filantrópicas (Anexo).

$2^{\circ}$ ano: Diagnóstico, planejamento e intervenção em saúde, alimentação e nutrição: A atividade prática do segundo ano do Curso também é realizada nos mesmos territórios com o propósito de complementar o diagnóstico e de realizar um planejamento estratégico situacional. $\mathrm{Na}$ terceira fase os estudantes aplicam inquéritos socioeconômicos e alimentar, avaliam o estado nutricional dos moradores e realizam levantamento de dados nos locais de comercialização de alimentos de referência para habitantes do território. Na quarta fase os estudantes elaboram um diagnóstico com as informações coletadas nos três semestres anteriores para o planejamento estratégico situacional. Ao final do semestre, os estudantes apresentam aos coordenadores de distritos sanitários, bem como aos coordenadores e aos nutricionistas dos CSF todas as etapas que compõe o planejamento, com enfoque na planificação de ações em saúde, alimentação e nutrição (Anexo).

- $3^{\circ}$ ano: Promoção da saúde em alimentação e nutrição: A atividade prática prevista para o terceiro ano do Curso implica na realização de uma intervenção nos territórios de saúde. Na sexta fase as ações estão relacionadas com as disciplinas Nutrição nos Ciclos de Vida e Cálculo e Análise de Dietas para Indivíduos. Na quinta fase os estudantes são incentivados a planejar e executar ações relacionadas à promoção da saúde em coletividades em conjunto com os estagiários da área de Nutrição em Saúde Pública (Anexo).
- 4ano: Ações de alimentação e nutrição na prevenção, tratamento e recuperação da saúde: No quarto ano há uma intersecção com a formação na área da Saúde Coletiva nas disciplinas de Ética e Bioética e Atenção e Assistência Alimentar e Nutricional. Pelo fato das atividades práticas estarem concentradas no nível de alta complexidade da atenção à saúde do SUS, há uma descontinuidade da formação voltada especificamente para a área de Nutrição em Saúde Coletiva (Anexo).

- 5० ano: O exercício profissional em Nutrição em Saúde Coletiva: Na disciplina de Estágio Supervisionado de Nutrição em Saúde Pública os estudantes desenvolvem ações de promoção, manutenção e recuperação da saúde no território em saúde, incluindo as Redes de Atenção Básica e de ensino público do município de Florianópolis (SC), de maneira articulada com os nutricionistas e demais profissionais de tais redes. São desenvolvidas ainda ações locais e intersetoriais de acordo com a demanda apontada pelos profissionais de saúde, podendo ocorrer em instituições sociais, de controle social, feiras, sacolões, entidades filantrópicas, entre outras. Além disso, ocorre atendimento nutricional tanto em nível ambulatorial como em grupos terapêuticos, entre outras atividades (Anexo).

Essa organização curricular apresenta desafios a serem superados que serão discutidos na seção que segue.

\section{Desafios para a formação em Nutrição em Saúde Coletiva na UFSC}

Entre os avanços alcançados com o currículo implantado em 2009 destacam-se: 1) A inserção na atenção básica do SUS ${ }^{6,14,17,24}$ desde a primeira fase do Curso; 2) A integração das diversas áreas da Nutrição (Nutrição Clínica, Nutrição em Unidade de Alimentação e Nutrição, Nutrição em Saúde Coletiva e Nutrição Básica e Experimental) ${ }^{25,35-38}$, em várias disciplinas; 3) 0 desenvolvimento de ações intersetoriais $5^{6,35-38 ; ~ 4) ~} 0$

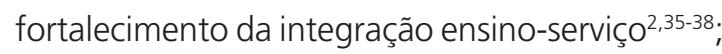


e 5) A inclusão de temas contemporâneos para a formação do nutricionista em Saúde Coletiva, como a SAN ${ }^{23,32}$, sistemas alimentares e o respeito à cultura ali-mentar6,12,18,33.

Apesar disso, são percebidos desafios que precisam ser transpostos, tanto no espaço da universidade, quanto nos cenários de prática.

O primeiro deles é a mudança de paradigma referente ao processo educativo, imperativa para a inserção dos docentes em novos cenários de prática ${ }^{2}$. Nesse sentido, parece fazer parte do cotidiano dos professores da Saúde Coletiva a atuação na atenção básica. No entanto, nas disciplinas interáreas, que contam com a participação de professores das demais áreas da Nutrição percebe-se certo desconforto de docentes, o que pode revelar a necessidade de educação permanente para os docentes do Curso de Nutrição e demais Cursos que compõem a área da saúde. Esta necessidade parte do pressuposto de que a inserção de discentes e docentes nos diversos cenários de prática e em seu contexto social ${ }^{18}$, independente da área de conhecimento, possibilita uma atuação capaz de promover a corresponsabilização e protagonismo dos sujeitos, necessária para atuação no SUS2,6,14,17,24,36,38.

Ao abordar sobre a formação pedagógica de professores de Nutrição, Costa ${ }^{2}$ aponta que as resistências por parte de docentes às mudanças exigidas nas DCN de 2001',8 estão relacionadas com a desvalorização das atividades de ensino e na formação discente não focada no aprender a aprender. Outro aspecto percebido é que o ensino desenvolvido por meio de metodologias ativas ${ }^{35-38}$ esbarra na inadequação da estrutura física das salas de aula, impedindo ou limitando seu desenvolvimento. Outros aspectos limitantes são a estrutura departamental da universidade, a não flexibilização de horários de aulas, o choque de horários entre os professores para participação em reuniões de fase e a organização disciplinar do Curso ${ }^{35-38}$, os quais limitam a atuação integrada entre as disciplinas de uma mesma fase e entre as fases, comprometendo a articulação entre os conteúdos abordados nas disciplinas. Outro fator recorrente é a percepção por parte dos discentes de que há repetição de conteúdos, pois os eixos transversais (Promoção da Saúde, SAN e DHAA) norteiam as discussões de diversas disciplinas.

Ao mesmo tempo foi identificado que há o limite da própria formação, relacionado à carga horária mínima do Curso definida pelo Conselho Nacional de Educação 1,7,8,39, que impossibilita trabalhar com todos os conteúdos apontados pelos estudantes como necessários. Uma das alternativas é possibilitar ao discente autonomia na busca do conhecimento, maior disponibilização de disciplinas optativas e de atividades complementares $^{8}$. No entanto, destaca-se a necessidade de aprofundamento no Curso das próprias orientações das DCN ${ }^{8}$ que não estão centradas na carga horária, mas sim no delineamento do perfil do egresso e nas competências e habilidades necessárias para entender e atuar diante das necessidades de saúde da população ${ }^{3}$.

No dia a dia dos professores constata-se dificuldade de acompanhamento tutorial do estudante, havendo, inclusive, um número reduzido de avaliações individuais ao longo do semestre nas disciplinas articuladas. Ademais, o acompanhamento tutorial do trabalho em grupos com 10 a 12 estudantes também dificulta a avaliação da integração e entrosamento do próprio grupo, no tocante a aprender a conviver, sendo comum ao longo do Curso a mobilidade entre membros dos grupos. A mudança de território ao longo da formação pode acarretar em uma quebra do entendimento do território e no desconhecimento de alguns indicadores de saúde e alimentação (coletados no primeiro e parte do segundo ano do curso) ${ }^{18,24}$, com interferência direta no planejamento e desenvolvimento de ações que promovam à saúde e Segurança Alimentar e Nutricional da população (atividades realizadas no final do segundo ano).

A inserção mais intensa no território em saúde torna pujante a integração entre o ensino e o serviço e a reestruturação curricular revela a necessidade de incorporar a esse espaço de discussão os nutricionistas que atuam na vigilância 
sanitária e na atenção de média complexidade. Este desafio torna-se maior, pois no município de Florianópolis atualmente há ausência de nutricionista nesse nível de atenção e a formação na UFSC ainda apresenta essa lacuna.

A atuação dos nutricionistas da Rede nos moldes do NASF e com vínculo terceirizado na alimentação escolar compromete o envolvimento desses profissionais com a formação dos estudantes. Este profissional, por atuar em vários centros de saúde e unidades educativas, tem dificuldades de acom-panhar a atividade discente nos cenários de prá-tica, o que demanda orientação presencial de professores das disciplinas. Se por um lado, isto fortalece a relação dos docentes e discentes com a realidade social, por outro, limita a aproximação da prática realizada pelo estudante à da atuação dos nutricionistas. Há o entendimento de que esse limite pode ser debatido e, futuramente, superado. Algumas estratégias para esta superação estão centradas no próprio fortalecimento da interação ensinoserviço e ainda em mudanças curriculares que alinhem horários de prática com as ações dos nutricionistas, na perspectiva de superar a fragmentação do saber e reintegrar conhecimentos ${ }^{36}$.

No mesmo sentido, destaca-se a fragilidade da atuação integrada entre Nutrição e demais cursos da área da saúde. Observa-se que não há integração curricular dos estudantes da área da saúde. Somente na disciplina de estágio obrigatório há uma aproximação multiprofissional, mas restrita às atividades pontuais. Como exemplo, cita-se a realização de territorialização com estudantes de Medicina; desenvolvimento de ações no Grupo denominado Idoso Ativo com estudantes de Educação Física e a participação em atividades lúdicas nas campanhas de vacinação com estudantes de Enfermagem.

Ressalta-se que os avanços do Curso de Nutrição da UFSC são frutos de avaliações contínuas das práticas pedagógicas em seus diferentes fóruns, além das parcerias institucionalizadas da UFSC com as secretarias municipais de saúde e de educação, como mencionadas anteriormente. Destaca-se ainda que, apesar dos desafios apontados, com o apoio financeiro e técnico-operacional do Programa Nacional de Reorientação da Formação Profissional em Saúde (Pró-Saúde) ${ }^{23,30}$, a formação dos profissionais de saúde tem se revelado uma iniciativa favorável para o fortalecimento do diálogo e integração da relação ensino-serviço, consequentemente, para uma formação dos estudantes do Curso de Nutrição da UFSC mais comprometida com a resolução dos problemas da população̊.

\section{CONSIDERAÇÕES FINAIS}

As Diretrizes Curriculares Nacionais recomendam que a formação em nutrição no país seja reorientada para fortalecimento do SUS. O Curso de Nutrição da UFSC, desde a sua criação, tem apresentado elementos facilitadores para este processo de formação, tais como as estratégias de avaliações contínuas em fóruns de discussões e de deliberações e a inserção institucional da UFSC no estabelecimento de parcerias com o SUS. Aliado a este contexto, outras estratégias nacionais nortearam e tem dado apoio a esta formação, como o Pró-Saúde.

Ressalta-se como avanços obtidos: 1) a integração entre disciplinas e com docentes de diferentes áreas de conhecimento/atuação; 2) a inserção dos discentes nos distintos cenários de prática; 3) o envolvimento docente-discente com os profissionais da Rede; 4) o processo de discussão contínua de formação com base em eixos transversais como promoção da saúde, SAN e DHAA; e 5) o planejamento de ações de promoção da saúde pautado na realidade do território e da população. Apesar disto, o Curso de Nutrição da UFSC apresenta desafios a serem superados.

Os novos ajustes curriculares devem ser acompanhados de um processo de formação docente que continue incentivando mudanças de práticas pedagógicas tradicionais, avançando para práticas educativas que englobem a diversidade dos cenários de prática dentro de uma perspectiva crítica e reflexiva. 
Além disso, caracteriza-se como pertinente o fortalecimento da integração ensino-serviço para que as demandas, limitações e desafios estruturais da universidade e do serviço sejam compreendidos, e que avancem na perspectiva de fortalecimento da rede docente assistencial.

As experiências exitosas e novas pesquisas com base nas análises das DCN e de Projetos Pedagógicos dos Cursos de Graduação em Nutrição no Brasil devem ser incentivadas para possibilitar a avaliação da atuação do nutricionista no âmbito do SUS, bem como fomentar e contribuir com a formação permanente desses profissionais e futuros nutricionistas.

\section{COLABORADORES}

J DAS NEVES, AA SOUSA e FAG VASCONCELOS par-ticiparam de forma articulada e simultânea do planeja-mento, redação e revisão do manuscrito.

\section{REFERÊ NCIAS}

1. Brasil. Presidência da República. Lei n 9.394, de 20 de dezembro de 1996. Estabelece as diretrizes e bases da educação nacional. Diário Oficial da União. 1996; 23 dez [acesso 2014 abr 29]; p.27.833. Disponível em: <https://www.planalto. gov.br/ccivil_03/Leis/L9394.htm>.

2. Costa NMSC. Formação pedagógica de professores de nutrição: uma omissão consentida? Rev Nutr. 2009; 22(1):97-104. doi: 10.1590/S1415-527320 09000100009

3. Soares NT, Aguiar AC. Diretrizes curriculares nacionais para os cursos de nutrição: avanços, lacunas, ambiguidades e perspectivas. Rev Nutr. 2010; 23(5):895-905. doi: 10.1590/\$1415-527320100 00500019

4. Vasconcelos FAG. A ciência da nutrição em trânsito: da nutrição e dietética à nutrigenômica. Rev Nutr. 2010; 23(6):935-45. doi: 10.1590/S1415-52732 010000600001

5. Vasconcelos FAG, Calado CLA. Profissão nutricionista: 70 anos de história no Brasil. Rev Nutr. 2011; 24(4):605-17. doi: 10.1590/S1415-527320 11000400009

6. Recine $E$, Gomes RCF, Fagundes AA, Pinheiro ARO, Teixeira BA, Sousa JS, et al. A formação em saúde pública nos cursos de graduação de nutrição no
Brasil. Rev Nutr. 2012; 25(1):21-33. doi: 10.1590/ S1415-52732012000100003

7. Medeiros MAT, Amparo-Santos L, Domene SMA. Education of dietitian's in Brazil: Minimum clock hours of instruction for a bachelor's degree in nutrition. Rev Nutr. 2013; 26(5):583-93. doi: 10.15 90/\$1415-52732013000500009

8. Brasil. Ministério da Educação e Cultura. Resolução CNE/CES n ${ }^{\circ}$ 5, de 7 de novembro de 2001. Institui Diretrizes Curriculares Nacionais do Curso de Graduação em Nutrição. Diário Oficial da União. 2001 [acesso 2014 abr 29]; Seção 1; 9 nov, p.39. Disponível em: <http://portal.mec.gov.br/cne/arquivos/ pdf/CES05.pdf>.

9. Banduk MLS, Ruiz-Moreno L, Batista NA. A construção da identidade profissional na graduação do nutricionista. Interface: 2009; 13(28):111-20. doi: 10.1590/S1414-32832009000100010

10. Ojeda BS, Creutzberg M, Feoli AMP, Melo DS, Corbellini VL. Nursing, nutrition and physiotherapy students: Career choice. Rev Latino-Am Enferm. 2009; 17(3):396-402. doi: 10.1590/S0104-11692 009000300018

11. Goulart RMM, Banduk MLS, Taddei JAAC. Uma revisão das ações de nutrição e do papel do nutricionista em creches. Rev Nutr. 2010; 23(4):655-65. doi: 10.1590/S1415-52732010000400015

12. Freitas MCS, Minayo MCS, Fontes GAV. Sobre o campo da Alimentação e Nutrição na perspectiva das teorias compreensivas. Ciênc Saúde Colet. 2011; 16(1):31-8. doi: 10.1590/S1413-8123201 1000100008

13. Geus LMM, Maciel CS, Burda ICA, Daros SJ, Batistel S, Martins TCA, et al. A importância na inserção do nutricionista na Estratégia Saúde da Família. Ciênc Saúde Colet. 2011; 16(Supl. 1):797-804. doi: 10.1590/S1413-81232011000700010

14. Jaime PC, Silva ACF, Lima AMC, Bortolini GA. Ações de alimentação e nutrição na atenção básica: a experiência de organização no Governo Brasileiro. Rev Nutr. 2011; 24(6):809-24. doi: 10.1590/S1415-5 2732011000600002

15. Vasconcelos FAG, Batista Filho M. História do campo da Alimentação e Nutrição em Saúde Coletiva no Brasil. Ciênc Saúde Colet. 2011; 16(1):81-90. doi: 10.1590/S1413-81232011000100012

16. Camossa ACA, Telarolli Junior R, Machado MLT. O fazer teórico-prático do nutricionista na estratégia saúde da família: representações sociais dos profissionais das equipes. Rev Nutr. 2012; 25(1):89-106. doi: 10.1590/S1415-52732012000100009

17. Cervato-Mancuso AM, Tonacio LV, Silva ERd, Vieira VL. A atuação do nutricionista na Atenção Básica à Saúde em um grande centro urbano. Ciênc Saúde Colet. 2012; 17(12):3289-300. doi: 10.1590/S141 3-81232012001200014 
18. Pinheiro ARO, Recine E, Alencar B, Fagundes AA, Sousa JS, Monteiro RA, et al. Percepção de professores e estudante em relação ao perfil de formação de nutricionista em saúde pública. Rev Nutr. 2012; 25(5):632-43. doi: 10.1590/S1415-5273 2012000500008

19. Santos LAS. O fazer educação alimentar e nutricional: algumas contribuições para reflexão. Ciênc Saúde Colet. 2012; 17(2):455-62. doi: 10.1590/\$14 13-81232012000200029

20. Santos LAS, Paiva JB, Mello AL, Fontes GAV, Sampaio LR, Freitas MCS. O nutricionista no programa de alimentação escolar: avaliação de uma experiência de formação a partir de grupos focais. Rev Nutr. 2012; 25(1):107-17. doi: 10.1590/S1415-52 732012000100010

21. Albuquerque $A G$, Pontes $C M$, Osório MM. Knowledge of educators and dieticians on food and nutrition education in the school environment. Rev Nutr. 2013; 26(3):291-300. doi: 10.1590/S1 415-52732013000300004

22. Chaves LG, Santana TCM, Gabriel CG, Vasconcelos FAG. Reflexões sobre a atuação do nutricionista no Programa Nacional de Alimentação Escolar no Brasil. Ciênc Saúde Colet. 2013; 18(4):917-26. doi: 10.1590/S1413-81232013000400003

23. Vieira VL, Utikava N, Cervato-Mancuso AM. Atuação profissional no âmbito da segurança alimentar e nutricional na perspectiva de coordenadores de cursos de graduação em Nutrição. Interface. 2013; 17(44):157-70. doi: 10.1590/S1414-32 832013000100013

24. Mais LA, Domene SMA, Barbosa MB, Taddei JAAC. Diagnóstico das práticas de alimentação complementar para o matriciamento das ações na Atenção Básica. Ciênc Saúde Colet. 2014; 19(1):93-104. doi: 10.1590/1413-81232014191.2168

25. Brasil. Conselho Federal de Nutricionistas. Resolução CFN n 380/2005. Dispõe sobre a definição das áreas de atuação do nutricionista e suas atribuições, estabelece parâmetros numéricos de referência por área de atuação e dá outras providências. Brasília: CFN; 2005 [acesso 2014 abr 29]. Disponível em: <http://www.cfn.org.br/novosite/pdf/res/2005/ res380.pdf $>$.

26. Santos LAS, Silva MCM, Santos JM, Assunção MP, Portela ML, Soares MD, et al. Projeto pedagógico do programa de graduação em nutrição da Escola de Nutrição da Universidade Federal da Bahia: uma proposta em construção. Rev Nutr. 2005; 18(1):105-17. doi: 10.1590/S1415-52732005000100010

27. Alves $E$, Rossi CE, Vasconcelos FAG. Nutricionistas egressos da Universidade Federal de Santa Catarina: áreas de atuação, distribuição geográfica, índices de pós-graduação e de filiação aos órgãos de classe. Rev Nutr. 2003; 16(3):295-304. doi: 10.1590/ S1415-52732003000300007
28. Vasconcelos FAG, Rossi CE, Costa M. Evolução histórica do nutricionista em Santa Catarina (1980-2003). Rev Ciênc Saúde. 2005; 24(1/2):28-35.

29. Universidade Federal de Santa Catarina. Pró-Reitoria de Ensino de Graduação: acompanhamento de egressos. Egressos da UFSC. Florianópolis: UFSC [acesso 2014 nov 2]. Disponível em: <http://www. egressos.ufsc.br/listaEgressos.xhtml>.

30. Brasil. Ministério da Saúde. Ministério da Educação. Programa Nacional de Reorientação da Formação Profissional em Saúde - Pró-Saúde: objetivos, implementação e desenvolvimento potencial. Brasília: Ministério da Saúde; 2009 [acesso 2014 abr 30]. Disponível em: <http://bvsms.saude.gov.br/bvs/ publicacoes/programa_nacional_reorientacao_ profissional_saude.pdf>.

31. Reibnitz KS, Daussy MFS, Silva CAJ, Reibnitz MT, Kloh D. Rede docente assistencial UFSC/SMS de Florianópolis: reflexos da implantação dos projetos Pró-Saúde I e II. Rev Bras Educ Med. 2012; 36(1):6875. doi: 10.1590/S0100-55022012000300011

32. Maluf R. Segurança alimentar e nutricional. Petrópolis: Vozes; 2007.

33. Valente FLS. O direito humano à alimentação: desafios e conquistas. São Paulo: Cortez; 2002.

34. Universidade Federal de Santa Catarina. Pró-Reitoria de Ensino de Graduação. Currículo do curso de bacharelado em Nutrição 2009. Florianópolis: UFSC; 2009 [acesso 2014 abr 30]. Disponível em: <http://www.nutricao.ufsc.br/files/2011/09/ CURRICULO_NUTRI\%C3\%87\%C3\%830_200913.pdf 3.pdf>.

35. Berbel, NAN. A problematização e a aprendizagem baseada em problemas: diferentes termos ou diferentes caminhos? Interface. 1998; 2(2):139-54. doi: 10.1590/S1414-32831998000100008

36. Barbosa MA, Brasil VV, Sousa ALL, Monego ET. Refletindo sobre o desafio da formação do profissional de saúde. Rev Bras Enferm. 2003; 56(5):574-6. doi: 10.1590/S0034-71672003000500022

37. Bordenave J, Pereira A. A estratégia de ensino aprendizagem. 26ª ed. Petrópolis: Vozes; 2005.

38. Limberger JB. Metodologias ativas de ensino-aprendizagem para educação farmacêutica: um relato de experiência. Interface: Comunic Saúde Educ. 2013; 17(47):969-75. doi: 10.1590/1807-576 22013.3683

39. Brasil. Ministério da Educação e Culutra. Resolução CNE/CES n 4, de 6 de abril de 2009. Diário Oficial da União. 2009 [acesso 2014 nov 3]; 7 abr; Seção 1:27. Disponível em: <http://portal.mec.gov.br/ dmdocuments/rces004_09.pdf>.

Recebido em: 7/5/2014 Versão final em: 6/11/2014 Aprovado em: 12/11/2014 
ANEXO

RELAÇÃO DE DISCIPLINAS INTEGRANTES DO CURSO DE NUTRIÇÃO DA UFSC QUE POSSIBILITAM O CUMPRIMENTO DAS HABILIDADES E COMPETÊNCIAS DA ATUAÇÃO DO NUTRICIONISTA EM SAÚDE COLETIVA.

Disciplinas $\quad$ Ementas

$1^{\circ}$ ano (primeira fase): O território em Saúde, Alimentação e Nutrição

Educação em Saúde, Alimentação e Nutrição I

Contextualização histórica de território em alimentação e nutrição. Relação áreas de conhecimento em nutrição e território. Direito humano à alimentação adequada para a promoção da Saúde e Segurança Alimentar. Conceito, compreensão e significado de território e territorialização. O território em saúde, alimentação e nutrição.

Desenvolvimento da Comunidade

Cultura, Consumo e Padrões Alimentares

Sociologia e Saúde

Metodologia Científica

$1^{\circ}$ ano (segunda fase): O território e o diagnóstico em Saúde, Alimentação e Nutrição alimentação brasileira. Marginalidade social e luta de classes.

O processo saúde e doença. Saúde como construção social. História das políticas de Saúde do Brasil. Educação e Promoção à Saúde. Introdução ao pensamento estratégico em saúde. A Nutrição e a Atenção Primária.

Processo de construção dos saberes referentes à história da alimentação e sua relação com a cultura e soberania alimentar dos povos. Influências culturais na

Evolução do pensamento sociológico. Contexto histórico do surgimento da Sociologia. Correntes do pensamento social: estruturalismo, funcionalismo e materialismo. Classes urbanas e rurais. Classes, poder e Estado no Brasil.

Técnicas de Pesquisa bibliográficas. Normalização do trabalho científico.

Evolução histórica da concepção de saúde e estágios evolutivos das profissões de saúde. Evolução histórica da organização dos serviços de saúde e níveis de aplicação das medidas preventivas (conceitos e atividades de saúde pública). O saneamento no processo de urbanização e sua determinação no estadode saúde. Água, esgoto e lixo. Condições locais e usos decorrentes. Medidas de tratamento e proteção.

Bioestatística II

Noções básicas de Bioestatística, levantamento e apuração de dados em saúde, análise descritiva de dados, noções de probabilidade.

Epidemiologia Básica

Aspectos históricos da epidemiologia e historicidade dos conceitos e usos da epidemiologia. Conceituação de saúde e doença. Causalidade e determinação do processo saúde/doença. Medidas de mortalidade e Morbidade. Método epidemiológico. Investigação epidemiológica. Conceito de risco.

Educação em Saúde, Alimentação e Nutrição ॥

Introdução às Políticas Públicas de Alimentação e Nutrição. O Sistema de Segurança Alimentar e Nutricional (SISAN) nas distintas esferas administrativas: municipal, estadual e federal. Identificação dos programas de alimentação e nutrição nos distintos territórios de prática.

Produção, Distribuição e Aquisição de Alimentos

Produção, distribuição e aquisição de alimentos; compreensão da cadeia produtiva alimentar, desde a origem ao consumo. Identificação dos sistemas agroalimentares e os diferentes modelos produtivos, relacionando-os aos alimentos com suas diferentes especificidades.

$2^{\circ}$ ano (terceira fase): Diagnóstico em Saúde, Alimentação e Nutrição

Epidemiologia nutricional

Magnitude dos distúrbios nutricionais em nível mundial e no Brasil. Epidemiologia dos distúrbios nutricionais: carências nutricionais específicas e doenças por excesso. Transição epidemiológica e nutricional. Estudo e aplicação dos métodos de inquéritos de consumo alimentar e sócio-econômico. Conceito e construção do Sistema de Vigilância Alimentar e Nutricional (SISVAN). SISVAN como conceito ampliado de vigilância em saúde (consumo alimentar, sócioeconômico, dados antropométricos, demográficos, epidemiológicos, sanitários). Análise da estratégia Global para alimentação, atividade física e saúde da Organização Mundial de Saúde (OMS). 


\section{ANEXO}

RELAÇÃO DE DISCIPLINAS INTEGRANTES DO CURSO DE NUTRIÇÃO DA UFSC QUE POSSIBILITAM O CUMPRIMENTO DAS HABILIDADES E COMPETÊNCIAS DA ATUAÇÃO DO NUTRICIONISTA EM SAÚDE COLETIVA.

Disciplinas Ementas

$2^{\circ}$ ano (terceira fase): Diagnóstico em Saúde, Alimentação e Nutrição

Métodos de diagnóstico nutricional individual e coletivo: métodos antropométricos, clínicos, bioquímicos, dietéticos e sócio-econômicos-culturais, nos diferentes ciclos de vida (gestantes, nutrizes, lactentes, pré-escolares, escolares, adolescentes, adultos e idosos). Conceito e construção do sistema de vigilância do estado nutricional de comunidades e indivíduos.

Vigilância Sanitária Alimentar (VISA)

Discussão de políticas públicas e segurança alimentar: Lei Orgânica de Segurança Alimentar e Nutricional (LOSAN) e o Sistema de Segurança Alimentar e Nutricional (SISAN). O papel do Conselho Nacional de Segurança Alimentar e Nutricional (CONSEA). Discussão da atuação de nutricionistas frente à qualidade dos alimentos disponíveis para a aquisição e consumo da população e a responsabilidade dos serviços municipal, estadual e federal de Vigilância Sanitária de Alimentos, em defesa da saúde coletiva.

$2^{\circ}$ ano (quarta fase): Planejamento em Saúde, Alimentação e Nutrição

Planejamento e Programação de Ações em Tipos e correntes de planejamento existentes: administrativo, normativo, esAlimentação e Nutrição

tratégico, estratégico situacional. Diagnóstico e avaliação das políticas públicas de alimentação e nutrição a nível federal, estadual e municipal nos distintos territórios de prática. Planejamento de ações de intervenção em alimentação e nutrição.

$3^{\circ}$ ano (quinta fase): Açães de promoção em Alimentação e Nutrição para diferentes ciclos de vida

Nutrição nos Ciclos de Vida

Estudo das necessidades e recomendações de energia e nutrientes nos diferentes ciclos de vida (gestantes, nutrizes, lactente, pré-escolar, escolar, adolescentes, adultos e idosos), visando à promoção da saúde. Orientação e atendimento em instituições, centros comunitários, associações de moradores para os diferentes grupos.

$3^{\circ}$ ano (sexta fase): Alimentação e Nutrição: ações de planejamento e de intervenção para os diferentes grupos

Nutrição em Saúde Pública

Histórico da atuação do nutricionista em saúde pública e sua atuação nos diferentes níveis de organização dos serviços de saúde; promoção e prevenção em alimentação e nutrição nos níveis de atenção em saúde; concepções de educação e a prática de educação alimentar e nutricional; intervenção em alimentação e nutrição em saúde pública.

Psicologia e Nutrição

Conhecimentos básicos em psicologia. História da Psicologia. Psicologia e Território em Saúde Mental. O modelo CAPS. Concepções do normal versus patológico. Aspectos biopsicossociais envolvidos no comportamento alimentar. Transtornos alimentares. Psicologia Social: uma visão de prevenção e promoção da saúde. Interação humana e relações interpessoais: aspectos interatuantes na relação do nutricionista com o paciente (cliente e família).

$4^{\circ}$ ano (sétima e oitava fases): Alimentação e Nutrição nas ações de prevenção, tratamento e recuperação da saúde

Ética e Bioética

Ética da qualidade de vida humana: pré-condições para a vida e a prosperidade humana.

Atenção e Assistência Alimentar e Nutricional Humanização da Atenção na Alimentação e Nutrição Hospitalar e Domiciliar.

$5^{\circ}$ ano (nona e décima fases): $O$ exercício profissional em três áreas de atuação do nutricionista

Estágio Supervisionado de Nutrição em Saúde Pública Capacitar estudantes do curso de Graduação em Nutrição, através da prática de estágios de Nutrição em Saúde Pública, que podem ser desenvolvidas em instituições de educação e de Saúde, ou outras instituições sociais, e em projetos de Extensão Comunitária, ressaltando a importância do domínio das técnicas disponíveis e de articulação efetiva com equipes interdisciplinares e organizações comunitárias. 
